\title{
UNDERSTANDING THE PATIENT'S PERSPECTIVE ON RAPID AND ROUTINE HIV TESTING IN AN INNER-CITY URGENT CARE CENTER
}

\author{
Angela B. Hutchinson, Giselle Corbie-Smith, \\ Stephen B. Thomas, Sveta Mohanan, and Carlos del Rio
}

\begin{abstract}
The purpose of this study was to explore patient perspectives of rapid and routine HIV-testing in an urgent care center at an urban public hospital. We conducted structured focus groups during a clinical trial comparing routinely offered rapid HIV-testing, routinely offered enzyme immunoassay (EIA) testing, and conventional EIA testing. Participants of the six focus groups were $89 \% \mathrm{Af}-$ rican American, $60 \%$ uninsured, and had a low educational status. Four independent coders analyzed the data using iterative content analysis. Rapid testing was preferred to EIA testing because it reduced the need for a return visit and stress of waiting for test results, though there were concerns about accuracy. Participants supported routinely offering testing, but there were concerns about privacy and cost. Fear and stigma were common reasons for refusing testing and not returning for results. Distrust and misconceptions about HIV, particularly regarding the importance of testing, were very common.
\end{abstract}

Lack of HIV counseling and testing (HIV CT), failure to receive HIV test results once tested, and late or delayed testing are significant obstacles to HIV prevention in the United States. An estimated one fourth of the 900,000 people in the United States infected with HIV do not know they are infected (Fleming et al., 2002). HIV-positive persons who do not know their serostatus miss the opportunity to undergo counseling to reduce high-risk behavior and prevent the transmission of HIV to others as well as the opportunity for early entry into care that is associated with improved prognosis (Palella et al., 2003). In addition, late testing, defined as progression to AIDS within 1

\footnotetext{
Angela B. Hutchinson was with the Grady Memorial Hospital, Georgia State University, and Georgia Institute of Technology, School of Public Policy at the time of this study. Giselle Corbie-Smith is with the University of North Carolina at Chapel Hill. Stephen B. Thomas is with the University of Pittsburgh. Sveta Mohanan is with the Carolinas HealthCare System. Carlos del Rio is with the Emory University School of Medicine.

This study was funded by the U.S. Centers for Disease Control and Prevention through Cooperative Agreement No. UR3/CCU416463. The authors thank the late Anna Dixon of Macro International Inc. for moderating the focus groups and Gloria Mejia for assistance with references. Additionally, we thank Ann Bostrom, Paul Farnheim, David Holtgrave, Angela Kim, and two anonymous reviewers for their comments. Angela Hutchinson is currently with the Centers for Disease Control and Prevention, Division of HIV/AIDS Prevention, Prevention Research Branch.

Address correspondence to Angela B. Hutchinson, Ph.D., M.P.H., Centers for Disease Control and Prevention, 1600 Clifton Rd., MS E-37, Atlanta, GA 30333; e-mail: ahutchinson@cdc.gov.
} 
year of diagnosis, is also problematic, particularly among minority populations, heterosexuals, and persons with low educational status (Centers for Disease Control and Prevention [CDC], 2003a). New CDC initiatives such as the serostatus approach to fighting the epidemic and advancing HIV prevention emphasize the need to increase HIV-testing and link HIV-infected persons to treatment and care (CDC, 2003b; Janssen et al., 2001). These initiatives include strategies to make HIV-testing a routine part of medical care and the use of rapid HIV-testing to increase the number of persons who learn their HIV status.

Conventional HIV CT involves offering HIV-testing based on risk factors and/or symptoms and testing using the enzyme-linked immunoassay (EIA) test. Clients tested using EIA must return to the testing center, typically in 1 to 2 weeks, for test results and posttest counseling. Positive tests are followed by Western blot, a high-specificity confirmatory test. Rapid testing is an alternative testing technology for which test results are available the same day, so in most cases clients learn their HIV status in the first visit (CDC, 1998). Clients who test positive on an initial rapid test are tested with Western blot; however, they are told their "preliminary positive results" and receive some posttest counseling. Similar to EIA, clients must return to the testing center for confirmatory test results and additional posttest counseling. Routine HIV CT is an alternative in which clients (typically aged 15-54) are routinely offered testing in populations with a high (greater than 1\%) prevalence of HIV (CDC, 1993; CDC, 2001a). This approach is advantageous because clients may not divulge risk factors and/or clinicians may not recognize HIV risk factors or have time to offer testing (Boekeloo et al., 1991; Kellock \& Rogstad, 1998).

Inner-city hospitals, particularly emergency departments and urgent care centers where undiagnosed HIV is problematic, are particularly suited for routine testing. Previous anonymous serprevalence surveys conducted in emergency departments report rates of undiagnosed HIV well above $1 \%$ and as high as $13 \%$, and approximately one fourth of patients with undiagnosed HIV had no identifiable risk factors (Alpert, Shuter, DeShaw, Webber, \& Klein, 1996; Goggin, Davidson, Cantrill, O’Keefe, \& Douglas, 2000; Kelen, Hexter, \& Hensen, 1995). For many patients, including those with undiagnosed HIV, emergency departments and urgent care centers serve as a primary source of health care and thus the only opportunity for HIV CT (Grumbach, Keane, \& Bindman, 1993; Kelen, Johnson, Digiovannan, Loring, \& Sivertson, 1990; Rask, Williams, Parker, \& McNagny, 1994). Unfortunately, HIV-testing has rarely been encouraged in emergency departments, even for patients with sexually transmitted diseases (Fincher-Mergi et al., 2002). Accordingly, many inner-city hospitals have begun implementing HIV screening programs to increase testing in emergency departments and urgent care centers. Several published studies report that HIV CT programs that have used routinely offered rapid HIV-testing in emergency departments and urgent care centers are highly effective and cost-effective at increasing testing and knowledge of HIV serostatus (CDC, 2001b; Hutchinson \& Farnham, 2003; Kelen, Shahan, Quinn, \& the Project Educate Work Group, 1999; Kroc et al., 2002; \& Kroc et al., 2003; Rothman, Ketlogetswe, Dolan, Wyer, \& Kelen, 2003; Walensky et al., 2003).

Though HIV screening programs that use routinely offered rapid HIV-testing in urgent care centers and emergency rooms have been shown to be effective, evidence of the patient's perspective must also be considered to understand how they are impacted and to facilitate successful implementation. It is particularly important to understand how patients view these newer HIV-testing strategies in emergency departments and urgent care centers given the urgent and episodic nature of the health problems for 
which the patients are presenting. For example, it would be important to know that patients do not find it stressful to make the decision to undergo testing given the urgent nature of their medical problems as well as that the patients do not feel pressure to consent to testing given all patients are being offered testing. Although there has been some exploration of patient acceptance of rapid testing in emergency departments, we are not aware of an in-depth qualitative assessment of routine testing and rapid testing in an urban urgent care center. The objective of this study, therefore, was to explore the patient's perspective of routinely offered HIV-testing and rapid HIV-testing as well as factors that influence HIV-testing (e.g., knowledge and beliefs) in an inner-city urgent care center serving a primarily African American patient population.

\section{METHOD}

This formative evaluation uses structured focus groups to gain insight into patient acceptability and preferences for HIV-testing and related testing behaviors such as returning for HIV test results. We chose focus groups because of the ability to facilitate discovery of a target population's knowledge and attitudes regarding health issues. Focus group interviews included questions about the acceptability and preferences for rapid and routine testing and reasons to accept or refuse testing and return for test results. We also asked general questions about HIV, including knowledge and risk perceptions, information sources and trust as these have the potential to influence testing behavior (Stein \& Nyamathi, 2000).

\section{DESCRIPTION OF STUDY SITE}

The focus groups were conducted during the formative evaluation phase of a larger clinical trial that compared three voluntary HIV-testing strategies: routinely recommend HIV-testing using rapid testing (SUDS, Abbott Laboratories), routinely recommended testing using EIA that required a return visit 2 weeks later for test results and 3) targeted HIV-testing using EIA (CDC, 2001b). The study was conducted in the Urgent Care Center of an urban public hospital serving the indigent population of Atlanta, Georgia. The Urgent Care Center is a walk-in medical clinic that also serves patients triaged from the hospital's emergency department. Accordingly, we perceived it to be better suited to HIV screening than the hospital's level one emergency department. HIV/AIDS is the second leading discharge diagnosis among adults at this institution, accounting for approximately $5 \%$ of all hospital discharges. This rate is well above the 1 per 1,000 that the CDC used as a cutoff in 1993 to recommend routine HIV-testing among hospitalized patients (CDC, 1993). At the time of the study, the Urgent Care Center was open 7 days a week for a total of 80 hours per week and had an average of 4,200 patient visits per month. The Urgent Care Center is also the sole source of health care for many patients; over $60 \%$ of patients in the hospital's Urgent Care Center or emergency department use these settings as their main source of health care (Rask et al., 1994). In addition, this patient population frequently encounters a lack of transportation, a lack of insurance and other financial barriers to accessing health care.

\section{FOCUS GROUP RECRUITMENT AND METHODS}

Focus group participants were recruited in the following manner (see Figure 1, schematic overview of focus group recruitment). HIV-testing was conducted during 1 -week periods of pilot testing the different testing strategies. During the 1st week, 


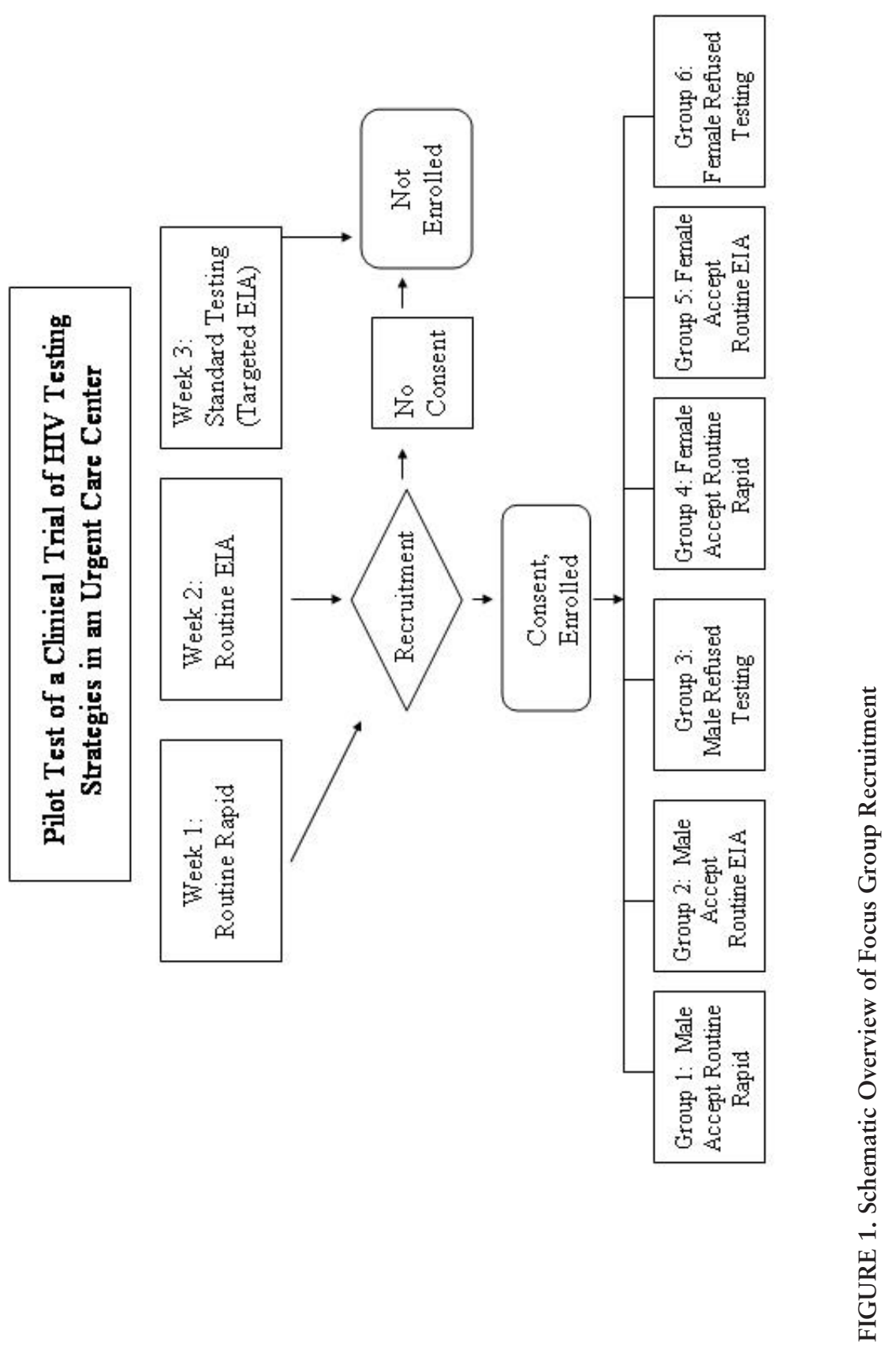


routine testing using the rapid test was offered; during the 2 nd week, routine testing using EIA was offered; and during the 3rd week, targeted EIA testing was offered. All patients who entered the clinic were offered an HIV educational brochure written at a sixth-grade reading level that described the type of HIV test offered that week. On routine testing weeks, clinicians were encouraged to offer HIV-testing to all patients aged 18-65 years who were not known to be HIV seropositive or tested during the preceding 6 months. Focus groups participants were recruited during each of the intervention weeks (routine rapid and routine EIA testing). After completion of the clinic appointment, patients were screened for participation in the focus groups, regardless of whether they had given their consent to HIV-testing. Ninety-two individuals were screened for participation, of whom 47 reported to the focus group session and participated. Group assignment was based on the type of HIV test taken, willingness to be tested and gender. There were six different focus groups; for each gender there was a group that (a) accepted routine rapid testing, (b) accepted routine EIA testing, and (c) refused testing. There was a $\$ 50$ incentive for participants who completed the focus group session. The Emory University Human Investigations Committee, the CDC Institutional Review Board, and the Grady Memorial Hospital Research Oversight Committee approved the study.

An African American professional moderator conducted the focus groups. The moderator's guide was divided by topic area and consisted of open-ended questions followed by a series of probes. Theories of behavioral decisionmaking were used to develop the focus group moderator's guide to provide a framework to evaluate how individuals perceive and evaluate alternative courses of action that included risk perception, problem structuring, outcome valuation as well as stage models of behavior change (Holtgrave, Tinsley, \& Kay, 1995). The focus groups interviews began with questions related to HIV knowledge and risk factors and moved to HIV-testing behaviors and then perceptions of HIV-testing methods. The focus group interviews were conducted at the hospital where the urgent care center is located. Informed consent was obtained at the beginning of each focus group and participants received a written copy of the consent form. The interviews were audiotaped and transcribed for subsequent content analysis.

\section{DATA ANALYSIS}

Four independent coders analyzed the data using iterative content analysis. Two of the coders were physicians with expertise in minority health and HIV/AIDS and two were social scientists with expertise in community health and health services research. These different perspectives provided important and diverse insights to the emerging results. For each iteration, each coder developed his/her own coding sheets using a combination of in vivo (literal terms used by the research subjects) and open codes (those that have been interpreted by the investigator) (Berg, 1998). Next, the coders met to establish agreement on the coding schemes and resolve differences that generally revolved around interpretation and context of the codes. Because agreement was established for each round of coding, we did not calculate interrater agreement statistics. During this first iteration, we focused on individual respondents to elucidate patterns of group dynamics. The primary coder used the individual analyses to develop a master record of the content analysis. In the next iteration, codes identifying the individual respondents were dropped so that group patterns were the focus of the coding. The objective of the final iteration was to further reduce the data by content areas and select quotations that represent themes, as well as outliers, within the con- 
tent domains. Four major content domains and 28 subdomains were identified. Any discrepancies between the coders were also resolved in this iteration. The primary coder then compared the results of the analysis with the transcripts to confirm validity of the content analysis.

\section{RESULTS}

\section{CHARACTERISTICS OF STUDY PARTICIPANTS}

Forty-seven individuals participated in the six focus groups with an average group size of eight (range $=5-11)$. There were slightly more male participants $(53 \%)$ than female $(47 \%)$. The characteristics of the focus group participants reflected the characteristics of the patient population. The participants were predominately African American (89\%); other races represented were white $(9 \%)$ and Asian/Pacific $(2 \%)$. The average age of the participants was 41 (range $=25-55)$. The demographics of the participants were similar to the general patient population of the hospital's Urgent Care Center and Emergency Care Center (Baker, Parker, Williams, \& Clark, 1998). The insurance status of the participants was as follows: $60 \%$ uninsured, $20 \%$ privately insured, $13 \%$ Medicaid, 4\% Medicaid/Medicare, and 2\% unknown. Finally, the participants had a relatively low educational status: $30 \%$ had less then a high school education, $40 \%$ had a high school education, $21 \%$ had some college, and only $9 \%$ had a college degree.

\section{RESEARCH FINDINGS}

We identified the following major content domains: HIV/AIDS knowledge, HIV information sources and trust, reasons for and against testing and returning for HIV test results, and preferences and acceptability of HIV-testing modalities (Table 1).

HIV/AIDS Knowledge. Focus group participants had very basic knowledge about HIV/AIDS. There was consensus that (a) HIV is the virus that causes AIDS, (b) there is no cure for AIDS but there are treatments to improve survival, and (c) HIV kills people by destruction of the immune system resulting in opportunistic infections. There was also awareness about prevention of HIV/AIDS. Interestingly, the only respondents to identify health education and awareness-raising efforts as a way to prevent HIV transmission were participants who refused to be tested. There were no differences by gender or testing group in terms of this basic HIV/AIDS knowledge.

Several misconceptions were identified that were equally distributed among the groups, for example, that AIDS is a cancer, airborne or related to personal hygiene. Participants were equally likely to name testing as a way to determine a person's HIV status as observing physical signs and symptoms. There were no meaningful differences by gender or testing group in this area. There was also considerable confusion around the concept of "incubation period" and "window period." For example, in a discussion of the incubation period for HIV, one participant said: "It might take 5 years to get to a point where the virus is detectable."

Most also agreed that retesting is important because there may be false-positive or false-negative results and because a person's status can change over time. Suggested intervals for retesting ranged from every other month to every year. Regarding HIV transmission, there was a greater focus on the low-risk exchange of bodily fluids as a means of transmission (including exchanging blood through open cuts/sores, saliva/kissing, and sweat) than types of high-risk sexual transmission (sexual intercourse, oral sex, and anal sex). Moreover, men were more likely than women to discuss the exchange of body fluids as a mode of transmission as were participants 
TABLE 1. Content Domains

\begin{tabular}{ll} 
Major Content Domains & \\
\hline $\begin{array}{l}\text { 1. HIV/AIDS knowledge } \\
\text { 2. HIV/AIDS information sources and trust }\end{array}$ & $\begin{array}{l}\text { 3. Reasons for and against testing and returning for test results } \\
\text { 4. Preference and acceptability of testing modalities }\end{array}$ \\
\hline $\begin{array}{l}\text { 1. What is HIV? } \\
\text { 2. Transmission }\end{array}$ & $\begin{array}{l}\text { 10. Good things about testing } \\
\text { 3. Prevention }\end{array}$ \\
$\begin{array}{l}\text { 11. Anonymity } \\
\text { 4. Treatment }\end{array}$ & $\begin{array}{l}\text { 12. Failure to return for test results } \\
\text { 5. Ways to know HIV status }\end{array}$ \\
$\begin{array}{l}\text { 6. Meaning of HIV test } \\
\text { 7. Incubation period }\end{array}$ & $\begin{array}{l}\text { 15. Routine testing } \\
\text { 16. Permission to be tested }\end{array}$ \\
9. Reasons for not testing & 17. Additional alternatives to testing \\
\hline
\end{tabular}

who refused testing, implying that these groups do have an awareness of this high-risk behavior. However, comments from respondents in all groups suggest a poor understanding of the concept "exchange of body fluids." For example, one participant said: "Anytime you go to a public place you come in contact with any kind of body fluids, I don't care whether it's saliva, blood or any kind of body fluid from another person that's infected, if it gets into an opening on you, then you have got it."

HIV Information Sources and Trust. Respondents were asked about preferred sources of information about HIV/AIDS. Overwhelmingly, health care providers and health care points of service (hospitals and health departments) were named as good sources for information. Traditional media outlets such as television, radio, and billboards were identified as preferred sources as some participants mentioned they preferred not to read information about HIV/AIDS. The Internet and public libraries were also popular because of the relative privacy afforded by those sources. The CDC was mentioned a source of "concrete information, not hearsay stuff" in two of the focus groups. In describing preferred sources, characteristics such as knowledge/expertise, trust, confidentiality, and convenience were mentioned. Several groups expressed the need for more information about HIV/AIDS ("You should see it everywhere, TV, bulletin boards, awareness groups"), though some participants pointed out that not everybody will be receptive to information about HIV/AIDS ("Some people just don't want to face the harsh reality that they could get AIDS").

Focus group participants were asked whether or not they trusted information they receive about HIV/AIDS. The male groups were the least trusting of information; however, in two of the groups, they trusted the information because they felt they "had to." One respondent expressed distrust in disclosure of HIV test results: "The doctor may not have been honest, they don't have the heart to tell them [they are HIV-positive]. ... Sometimes they put in on that piece of paper, but if you don't read that piece of paper, you don't know." Another common theme around distrust was the idea that the scientific community still doesn't know everything about the disease "like if it's carried in saliva or mosquitoes can transfer it ... they have no clue what they are talking about."

When discussing trust issues, conspiracy theories were raised in several of the focus groups and were more common in the male groups. Suggested conspirators in- 
clude "White people, pharmaceutical companies, doctors, and the government." One person talked about the possibility of a "wacky doctor injecting people with the AIDS virus such as a KKK [Ku Klux Klan] doctor." Another stated: "HIV comes from genocide and was probably invented during WWII." There were also perceptions about covert actions by the government, as expressed by the statement "There is a disease stronger than AIDS out there, man, and the government is taking everyone [with that disease] to an island and leaving them." Both male and female groups expressed the idea that there is a cure for AIDS. The government and pharmaceutical companies were thought to already have a cure for HIV/AIDS and were withholding it because of financial profits of current treatment. Another common theme is that there is a cure for HIV/AIDS that only rich people can afford.

Reasons for and Against Testing and Receiving Results. Reasons to accept HIV-testing fell into two general categories: those that were focused on risky behavior (high-risk behavior, concern about a partner's status, desire to prevent the spread of disease if infected) and those that were not (to determine status, to get treatment, to have peace of mind). Additionally, some women indicated that a reason to get tested was because "you don't trust your partner." It seems that participants view testing as beneficial even when personal behaviors that may put them at risk are not present. As one participant stated, "It's good to know where you stand." Finally, the fact that the test was free was the reason many participants were tested.

For all of the focus group participants, fear was the greatest single reason given for not accepting an HIV test when offered. Many different types of fear were mentioned and some were gender specific. Men were more likely to report a fear of dying, as well as a fear of the emotional or psychological consequences of testing positive such as anger, depression, guilt, and vengeance. One male participant stated; "If I got positive results, I am going to have thoughts about taking vengeance on the person I think gave it to me." Conversely, women were more likely to describe a fear of rejection from friends and family or being labeled as "gay or a drug user" as a reason to refuse testing. Across groups, concern about anonymity was also a reason for refusing testing. One respondent who refused testing stated that she was told to "never, ever get tested for HIV where they know your name and can get your medical records." Fear of employer discrimination was also expressed. Additional reasons included "irresponsibility" and "lack of loving oneself." Members of several of the focus groups said they would get tested if the test was more accurate as these participants had known someone who had a false positive test. Cost was also mentioned as a barrier, particularly for those at high risk. "A lot of high risk people have no money. They will buy a $\$ 10$ rock [crack] rather than pay for an HIV test," reported one participant.

The groups that refused testing were the only ones to identify convenience factors such as waiting for test results as a reason for not getting tested. Many patients had waited several hours to be seen and, in the case of the rapid test, did not want to wait an additional 2 hours. Those who refused testing also had more concerns about anonymity than the groups that accepted testing. This was evident in both their reasons for not getting tested and ways to improve testing. Additionally, they were the only groups that cited "not at risk" as a reason for not being tested. Other reasons for not getting tested include not wanting to change one's behavior and the stress of waiting for results. Finally, in the female group that refused testing, fear of becoming infected with HIV during testing was mentioned by several of the participants who discussed the need to "see them [the phlebotomist] take out a new [sterilized] needle." 
The reasons for not returning for test results were similar to the reasons for not getting tested for HIV. In nearly all of the focus groups, fear, loss of courage and inconvenience were common reasons for not returning for test results. As one respondent who did not return for HIV test results in the past suggested, "Ignorance is bliss." Another common theme was the fear of telling their partner they were HIV positive. In one group, the counseling process contributed to fear of being HIV-positive as evident by this quote: "When they tell you about HIV and why you might be at risk, you are too scared to know and come back." Additionally, as evident by one participant's comment- people do not return because they are shamefaced"—stigma associated with HIV-testing was also a barrier to returning for results. Inconvenience was more frequently mentioned in the female focus groups. Finally, some patients misunderstood the testing process; they stated that they assumed if the HIV test was positive and they did not return for their results, the health department would locate them: "If you tested positive, the health department would contact you anyway, right?" There was also the perception that they would receive certified letters in the mail if they were HIV-positive such as the case if an infectious disease were identified during blood donation. Additionally, in the case of anonymous testing, loss of a "secret number" was mentioned as a reason for not returning for test results.

Preference and Acceptability of Rapid and Routine Testing. The rapid HIV test was overwhelmingly described as preferable to the conventional EIA test based on the shorter turnaround time for test results. A common theme about rapid testing is that people preferred the rapid test because the additional wait time of the standard test is more stressful and might cause them to lose the courage to return for the test results. For example, one participant stated, "In a 2-hour period you could sit there or go to McDonald's, but if you have a whole week, that's planting stuff in your head." However, many of the female participants felt that the wait time would not affect their decision to get tested. Typical responses for people who did not express preferences for rapid testing included "I'm looking for results. It doesn't matter if it takes a day or a week, I just want to know" and "A test is a test. The decision is if you decide to take it.”

There was some concern about the accuracy of the rapid test among those who had received both types of tests. One person who refused testing stated: "What I want to know is how reliable is this test?" Others felt that they would feel more confident if it took longer to get the results. Another negative attitude about rapid testing that was expressed by the female group that received the rapid test was that it "was like an assembly line; it is just so impersonal." One member of the group stated that she didn't want to take the test but felt pressure because the doctor asked.

Although the rapid test offers same-day results, it extended the patient's total visit to the clinic by at least 2 hours. One patient commented: "The rapid test was excellent, even though it wasn't rapid." In several cases, participants who refused the rapid test stated they had waited up to 7 hours before they were seen and feared the additional 2 hours could lead to 4, 5, 6, or 7 more hours. Additionally, several participants who had refused the rapid test suggested that since the hospital was slow at processing patients, the rapid test should be offered when they arrive at the clinic before they are seen. Therefore, institutional barriers thwarted the innovation of rapid testing by making it less, rather than more convenient to some patients.

There was general support in all of the focus groups for routine testing. Those who supported the idea of routine testing felt that it was worthwhile and would "save more people." Another common theme was that if patients had considered testing and 
the doctor also suggested it, they would be willing to consent to testing. Likewise, participants felt that some people who need to be tested would not be tested unless it was routine. There was discussion in one group that routine testing is beneficial for those who have other chronic diseases because HIV/AIDS can aggravate these conditions. Some participants expressed concern that routine testing should not be done when you come in for common things like a cold, yet others felt that it should be done every time you go to the doctor "because most of the time people come to the doctor for an emergency." A few individuals stated that testing should be done only when HIV is suspected based on symptoms. One group suggested that routine HIV-testing should be mandatory at the state's expense and people should be required to have a card with their HIV status and most recent test date. In discussing routine testing, half of the groups stated that they would only support routine testing if it were voluntary. Of those who did not support routine testing, the biggest concern was that it was too expensive (for both the individual and the hospital) and it might compromise confidentiality.

We also assessed whether or not patients thought routine testing was already being done, for example, if they thought that they had been tested for HIV without their consent. In general, participants held the view that legally they had to give their permission to be HIV-tested; however, many were skeptical that this procedure was being followed. In all of the groups, participants believed that there are circumstances when patients are tested without consent such as when there is a trauma (so that doctors can protect themselves) or when a patient is a minor. In many cases, participants described situations when they had been tested for HIV without their consent. Another individual respondent said: "Doctors do what they want.... Y You don't know what is being checked on the lab sheet". Participants in one group believed that you have to consent to being tested for HIV before receiving any medical care at the hospital.

When asked about other attributes or testing methods that would increase the likelihood of a person agreeing to be tested, all of the focus groups named less invasive testing methods. Alternatives to drawing blood that were mentioned included saliva testing; finger stick testing; urinalysis; hair analysis; and skin, fingernail, stool, sweat, and tear samples. Among participants who refused testing, use of a home test was the most frequently reported suggestion for increasing testing rates.

\section{DISCUSSION}

By demonstrating patient acceptance of rapid and routinely offered HIV-testing, our study makes an important addition to the evidence of effectiveness and cost-effectiveness of these testing strategies in urgent care centers. This study also provides deeper insight and context to the quantitative finding of other studies. In our study, focus group participants who were patients of an urgent care center overwhelmingly preferred rapid HIV-testing to conventional EIA testing because it reduced the need for a return visit and the stress of waiting for results. They were also accepting of routinely offered HIV-testing and felt that more people would undergo testing with routine testing. Participants felt that less invasive testing such as saliva and finger-stick testing would increase the likelihood of being tested. Thus, it is likely that this patient population would prefer the more recently approved OraQuick rapid test because it is both less invasive (it uses serum samples obtained from a finger stick) and has a much shorter turn around time (20 minutes) than the SUDS rapid test (CDC, 2002). We found that fear was the primary reason for not undergoing testing and/or returning for HIV test results. Our findings also support previously reported research on HIV CT 
preferences in other at-risk populations (STD clinics, gay men, and injection drug users) that clients would be more willing to be tested if offered rapid testing, if less invasive testing methods were used and that fear is a significant barrier to testing (Spielberg, Kurth, Gorbach, \& Goldbaum, 2001). Our study adds to this literature by providing an in-depth exploration of routine testing and including an important at-risk population, minority patients that receive care at an urgent care center in an urban public hospital. This is a particularly important setting in terms of identifying undiagnosed HIV because urgent care centers frequently serve as the only source of health care and thus the only opportunity for diagnosis for this high-risk population (Rask et al., 1994). These data also offer insight into implementation of these testing strategies from a patient's perspective, particularly regarding pretest counseling, operational issues, and reasons for refusing testing and not returning for HIV test results

Although rapid and routine HIV-testing were accepted in this population, our results highlight factors that should be considered in implementing these testing strategies. Significantly, many participants did not feel confident about the accuracy and reliability of rapid tests, which has also been found in other populations (Greensides, Berkelman, Lansky, \& Sullivan, 2003). Given that rapid tests are comparable to EIA in terms of sensitivity and specificity, pretest and posttest counseling should explicitly address accuracy of rapid HIV tests (Kassler et al., 1995). Rapid testing was not preferred by those who waited several hours to be seen in the clinic before being offered the rapid test; thus providers should consider making rapid testing available to patients while they are waiting to be seen or during the actual clinic visit. Though focus group participants generally supported routine testing, they were uneasy about the confidentiality of routine testing programs and at least one participant expressed feeling pressure to consent to testing. Issues of confidentiality and voluntary consent should therefore be taken into consideration during counseling. Additionally, participants expressed concern over the cost of routine testing and many indicated that they would not undergo routine testing if they were required to pay for it. This is particularly concerning as the majority of these patients were without public or private health insurance.

Our findings revealed several misconceptions related to HIV-testing that should be considered in refining HIV CT programs. One is that idea that patients must consent to being tested for HIV before receiving any medical care at a hospital. Patients who hold this view could falsely believe that if they have received medical care at a hospital, then an HIV test was done and unless they are told otherwise, they can assume they are HIV-negative. Another misconception was that the health department would contact those who tested positive and failed to return for their test results. Pretest counseling should, therefore, specifically address the fact that even in states where HIV infection is reportable, the health department is not responsible for contacting individuals who test positive and do not return for their results. Additionally, many participants did not seem to understand the importance of being tested before physical symptoms are present and were focused on low-risk modes of transmission over high-risk sexual modes of transmission. This indicates that after the 2 nd decade of HIV disease, there is still a fair amount of misinformation and stigma. These factors might explain the high rates of late testing observed among minority populations and persons of low educational status (CDC, 2003a). In addition, our results demonstrate that conspiracy theories about HIV remain salient. These findings further the understanding of conspiracy theories and distrust among African American men that have 
previously been reported and emphasize the importance of culturally sensitive HIV prevention programs that address the issue of distrust (Corbie-Smith, Thomas, \& St. George, 2002; Klonoff \& Landrine, 1999).

We would like to acknowledge several limitations of our study. Because rapid testing was routinely offered, we did not have a focus group for persons tested with targeted rapid testing, so perceptions about rapid testing were expressed by those that had both testing strategies. We do not view this as a problem because all rapid testing programs that have been implemented in the emergency department and urgent care center setting routinely offered testing. Additionally, although we have gained insight into the target population's preferences for HIV-testing and counseling, we point out that the use of focus groups are not sufficient for elicitation of individual preferences. Finally, we point out that our findings are specific to our patient population and clinical setting, predominately low income, low educational status, inner-city African Americans in an urban public hospital. However, we point out that these population characteristics are common among those at highest risk for late HIV-testing (CDC, 2003a).

Given the formative nature of our study, we offer several suggestions for future research. Quantitative survey research should be conducted as next step in determining the generalizability of these findings to other patient populations and clinical settings and to further assess individual HIV-testing preferences. Additional studies are also needed to further understand operational issues associated with HIV CT in urgent care centers. Finally, although we have demonstrated that patients accept these testing methods, there is concern that they would not be willing to pay for them if they bore the cost; thus additional research is necessary to answer broader policy issues such as financing of emergency room HIV CT programs.

\section{REFERENCES}

Alpert P.L., Shuter J., DeShaw M.G., Webber, M.P., \& Robert, K. (1996). Factors associated with unrecognized HIV-1 infection in an iner-city emergency department. Annals of Emergency Medicine, 28(2), 159-164.

Baker, D.W., Parker R.M., Williams M.V., \& Clark W.S. (1998). Health literacy and the risk of hospital admission. Journal of General Internal Medicine, 13(12), 791-798.

Berg, B. (1998). Qualitative research methods for the social sciences (3rd ed.). Boston: Allyn \& Bacon.

Boekeloo, B. O., Marx, E. S., Kral, A. H., Coughlin, S. C., Bowman, M., \& Rabin, D. L. (1991). Frequency and thoroughness of STD/HIV risk assessment by physicians in a high-risk metropolitan area. American Journal of Public Health, 81(12), 1645-1648.

Centers for Disease Control and Prevention. (1993). Recommendations for HIV-testing services for inpatients and outpatients in acute care hospital settings. Morbidity and Mortality Weekly Reports, 42(RR-2), 157-158.
Centers for Disease Control and Prevention. (1998). Update: HIV counseling and testing using rapid tests-United States, 1995. Morbidity and Mortality Weekly Reports, 47, 211-215

Centers for Disease Control and Prevention. (2001 a). Revised guidelines for HIV counseling, testing, and referral. Morbidity and Mortality Weekly Reports, 50(RR19), 1-58.

Centers for Disease Control and Prevention. (2001b). Routinely recommended HIV-testing at an urban urgent care clinic-Atlanta, Georgia. Morbidity and Mortality Weekly Reports, 50, 538-541.

Centers for Disease Control and Prevention. (2002). Notice to readers: Approval of a new rapid test for HIV antibody. Morbidity and Mortality Weekly Reports, 2002, 51, 1051-1052.

Centers for Disease Control and Prevention. (2003a). Late versus early testing of HIV-16 sites United States, 2000-2003. Morbidity and Mortality Weekly Reports, 52, 581-586. 
Centers for Disease Control and Prevention. (2003b) Advancing HIV prevention: new strategies for a changing epidemic-United States, 2003. Morbidity and Mortality Weekly Reports, 52, 329-332.

Corbie-Smith, G., Thomas, S.B., \& St. George, D.M. (2002). Distrust, race, and research. Archives of Internal Medicine, 162(21), 2458-2463.

Fincher-Mergi, M., Cartone K.J., Mischler J., Pasieka P., Lerner E.B., \& Billittier A.J. (2002). Assessment of emergency department health care professionals' behavior regarding HIV-testing and referral for Patients with STDS. AIDS Patient Care and STDS, 16(11), 549-553.

Fleming, P., Byers, R.H., Sweeney, P.A., Daniels, D., Karon, J.M., \& Janssen, R.S. (2002). HIV prevalence in the United States [Abstract]. Program and abstracts of the Ninth Conference on Tetroviruses and Opportunistic Infections. Seattle, WA.

Goggin, M.A., Davidson, A.J., Cantrill, S.V., O'Keefe, K., \& Douglas, J.M. (2000). The extent of undiagnosed HIV infection among emergency department patients: results of a blinded seroprevalence survey and a pilot HIV-testing program. Journal of Emergency Medicine, 19(1), 13-19.

Greensides, D.R., Berkelman, R., Lansky, A., \& Sulllivan, P.S. (2003). Alternative HIV-testing methods among populations at high risk for HIV infection. Public Health Reports, 118, 531-539.

Grumbach, K., Keane, D., \& Bindman, A. (1993). Primary care and public emergency department overcrowding. American Journal of Public Health, 83(3), 372-378.

Holtgrave, D.R., Tinsley, B.J., \& Kay, L.S. (1995). Encouraging risk reduction. A decision making approach to message design. In E. Maibach \& R. Parrott (Eds.), Designing health messages: Approaches from communication theory and public health practice (pp. 24-40, 304). Thousand Oaks, CA: Sage.

Hutchinson, A.B., \& Farnham, P.G. (2003, July 27-30). Cost-effectiveness of HIV-testing strategies in the emergency care/urgent care center setting. Paper presented at the 2003 National HIV Prevention Conference. Atlanta, GA.

Janssen, R.S., Holtgrave, D.R., Valdiserri, R.O., Shepherd, M., Gayle, H.D., \& De Cock, K.M. (2001). The serostatus approach to fighting the HIV epidemic: Prevention strategies for infected individuals. American Journal of Public Health, 91(7), 1019-1024.

Kassler, W.J., Haley, C., Jones, W.K., Gerber, A.R., Kennedy, E.J., \& George, J.R. (1995) Performance of a rapid, on-site human immunode- ficiency virus antibody assay in a public health setting. Journal of Clinical Microbiology, 33, 2899-2902.

Kelen, G.D., Hexter, D.A., \& Hansen, K.N. (1995). Trends in human immunodeficiency virus (HIV) infection among a patient population in an inner-city emergency department: Implications for emergency department-based screening programs for HIV infection. Clinical Infectious Diseases, 21, 867-875.

Kelen, G.D., Johnson, G., Digiovanna, T.A., Loring, K., \& Sivertson, K.T. (1990). Profile of patients with human immunodeficiency virus infection presenting to an inner-city emergency department: Preliminary report. Annals of Emergency Medicine, 19, 963-969.

Kelen, G.D., Shahan, J.B., Quinn, T.C., \& the Project Educate Work Group. (1999). Emergency department-based HIV screening and counseling: Experience with rapid and standard serologic testing. Annals of Emergency Medicine, 33(2), 147-155.

Kellock, D. J., \& Rogstad, K. E. (1998). Attitudes to HIV-testing in general practice. International Journal of STD/AIDS, 9(5), 263-267.

Klonoff, E. A., \& Landrine, H. (1999). Do blacks believe that HIV/AIDS is a government conspiracy against them? Preventive Medicine, 28(5), 451-457.

Kroc, K., Kendrick, S., Withum, D., Couture, E., Miller, S., Zagorski, B., Branson, B., \& Weinstein, R. (2002). Rapid HIV-testing in an emergency department. Paper presented at the 2002 National STD Prevention Conference (2002, March). San Diego, CA.

Kroc, K., Papiez, G.R., Parks, D.I., Couture, E.F., Roberts, R.R., Straus, H.E., Peeler, L.N., Kendrick, S.R., Branson, B.M., \& Weinstein, R.A (2003, July). Routine screening of emergency department (ED) patients using Oraquick HIV-1 Antibody Test. Paper presented at the 2003 National HIV Prevention Conference. Atlanta, GA.

Palella, F.J., Deloria-Knoll, M., Chmiel, J.S., Moorman, A.C., Wood, K.C., Greenberg, A.E. Holmberg, S.D., \& HIV Outpatient Study Investigators. (2003). Survival benefits of initiating antiretroviral therapy in HIV-infected persons in different CD4+ cell strata. Annals of Internal Medicine, 138, 320-326.

Rask, K. J., Williams, M. V., Parker, R. M., \& McNagny, S. E. (1994). Obstacles predicting lack of a regular provider and delays in seeking care for patients at an urban public hospital. Journal of the American Medical Association, 271(24), 1931-1933.

Rothman, R.E., Ketlogetswe, K.S., Dolan, T., Wyer, P.C., \& Kelen, G.D (2003). Preventive care in the emergency department: should emer- 
gency departments conduct routine HIV screening? A systematic review. Preventive Care, 10(3), 278-285.

Spielberg, F., Kurth, A., Gorbach, P.M., \& Goldbaum, G. (2001), Moving from apprehension to action: HIV counseling and testing preferences in three at-risk populations. AIDS Education and Prevention, 13(6), 524-540.

Stein, J.A., \& Nyamathi, A. (2000). Gender differences in behavioral and psychosocial predic- tors of HIV-testing and return for test results in a high-risk population. AIDS Care, 12, 343-356.

Walensky, R.P., Losina, E., Malatesta, L., Barton, G.E., O’Connor, C.A., McGuire, J.F., \& Freedberg, K.A. (2003, July). Routine HIV testing: High yield in urgent care centers. Paper presented at the the 2003 National HIV Prevention Conference, Atlanta, GA. 